\title{
Análisis procedimental y docu- mental de un nombramiento de mayordomo de propios durante el reinado de Fernando VI.
}

\author{
Manuel Salamanca López *
}

Artículo recibido:

13 de junio de 2011.

Artículo aceptado:

1 de agosto de 2011.

\section{RESUMEN}

Los mayordomos se encargaban de la gestión financiera del Concejo, llevando la contabilidad de los ingresos y gastos. Por lo que respecta a Madrid, tenemos noticias de su existencia en el capítulo CXIII del Fuero, aunque será en el siglo XV cuando se consolide como oficio de carácter unipersonal, nombrado de forma anual. En la segunda mitad del siglo XVI, el cargo se dividirá en dos, el mayordomo de los propios del Concejo y el mayordomo del pósito. Durante el reinado de Fernando VI, su designación se realizará conforme a la costumbre, si bien, en ocasiones, como es nuestro caso, se producían alteraciones en su génesis. En esa línea, analizaremos las vicisitudes acontecidas durante la elección de Antonio de Bengoa, traducidas en el

* Universidad Complutense de Madrid, España. mail ???????????

INVESTIGACIÓN BIBLIOTECOLÓGICA, Vol. 25, Núm. 54, mayo/agosto, 2011, México, ISSN: 0187-358X. pp. 159-181 
análisis del procedimiento y documentación generada a lo largo de su tramitación.

Palabras clave: Ferdinand VI, mayordomo de propios, Antonio de Bengoa, nombramiento, documentación.

\begin{abstract}
Procedural and documentary analysis of "mayordomo de propios" during the reign of Ferdinand VI Manuel Salamanca López

The "mayordomos" were responsible for the financial management of the Municipal Council, overseeing the accounting for income and expenditure. With regard to Madrid, we have news of your existence in the chapter CXXII of the "Fuero", although it will be in the 15th century, when it consolidated as office of unipersonal nature, appointed on an annual basis. In the second half of the 16th century, the office will be divided into two, the "mayordomo de los propios" of the Council and the "mayordomo del pósito". During the reign of Ferdinand VI, his nomination be made according to the custom, although, at times, as is our case, there were alterations in its genesis. In that line, we will analyze the vicissitudes occurred during the election of Antonio of Bengoa, translated into the analysis of the procedure and documentation generated during their processing.
\end{abstract}

Keywords: Fernando VI, mayordomo de propios, Antonio de Bengoa, appointment, documentation.

\title{
I. INTRODUCCIÓN
}

Z $\mathrm{n}$ opinión de Claudio Sánchez Albornoz, ${ }^{1}$ los inicios del cargo de mayordomo se remontan a tiempos de los reyes godos y hubo que esperar hasta la segunda mitad del siglo XII para encontrar un oficial similar en el 
ámbito municipal, momento a partir del cual el concilium o asamblea elegía anualmente un maiorinus, que se encargará de la gestión económica. ${ }^{2}$

Por lo que respecta a Madrid, en su Fuero se distinguen varios tipos de mayordomos, a saber, del Concejo, de fiadores, que ayudaban a la recolección de caloñas, y de la muralla. Aunque hay constancia de la existencia del oficio desde principios del siglo XIV, no tendremos noticias fehacientes de él hasta pasado un tiempo. ${ }^{3}$

Durante la Edad Moderna el cargo gana en consideración a la par que irán apareciendo nuevos textos reguladores (ordenanzas de Sevilla (1527), Libro verde de Segovia (1611), ordenanzas de Badajoz del siglo XVIII, etcétera).

Llegado el reinado de Fernando VI, los nombramientos de mayordomos de propios del Concejo de Madrid se harán conforme a las prácticas de carácter consuetudinario que habían llegado a un alto grado de normalización. Sin embargo, este precepto se verá alterado en nuestro caso por la sucesión de una serie de acontecimientos que afectarán al procedimiento y a su duración, no en vano las diligencias administrativas se extendieron a lo largo de cuatro años hasta su conclusión.

\section{Procedimiento administrativo}

\subsection{Información previa}

Eusebio de Sabugal, mayordomo de propios desde el año 1704, se jubiló en abril de 1744 tras 40 años de servicio, y falleció al poco tiempo. Este acontecimiento provocó la inmediata reacción del Marqués de Montealto, a la sazón corregidor de la villa de Madrid, quien, en virtud de un auto en forma acta de 12 de enero de 1745, dispuso que se designase nuevo mayordomo,

así para que recaude y exsija todas las rentas y caudales pertenezientes a ellos como para que se vaia entregando de todos los papeles pertenecientes a los referidos propios y conduzcan al recobro de sus rentas con toda expresión y claridad y que en ello haia la buena quenta y razón. ${ }^{4}$

2 Esteban Corral García, El mayordomo de Concejo en la Corona de Castilla (s.XIII-s.XVIII), Madrid: El Consultor de los Ayuntamientos y de los Juzgados, 1991, p. 35.

3 Rafael Gibert y Sánchez de la Vega, El concejo de Madrid. Su organización en los siglos XII al XV, Madrid, 1949, p. 241.

4 Archivo de Villa de Madrid (en adelante A.V.M.), Secretaría, 4-329-41. 
Al día siguiente, el citado Marqués hacía lo propio en el pleno del Concejo, determinando que trajeran los antecedentes de dicho asunto para disponer lo más conveniente. Semanas después se volvía a reunir el Consistorio resolvía que antes de hacer nombramiento alguno debía conocerse el estado en que se encontraba la contabilidad municipal. A este efecto, se llamó al contador Francisco Verdugo, quien se comprometió a fenecer las cuentas atrasadas de la citada mayordomía en un plazo no superior a tres meses, además de entregar en 15 días un extracto de todas ellas hasta fin de abril de 1744. Así, en razón de este hecho se suspendía la nominación de mayordomo de propios

y en el ínterin que es formalizado por dicho contador el citado estracto, la Junta de propios, a quien se comete, ejecuta un estado general de todos ellos con distinción de sus valores, cargas y consignaciones que tienen empeños y atrasos en que actualmente se hallan constituidos. Y hecho con la posible brevedad este manifiesto se trayga al ayuntamiento para que...pueda Madrid proceder a nombrar persona correspondiente para el uso exercicio y desempeño del mencionado empleo de mayordomo de dichos propios. ${ }^{5}$

Con todo, dicha elección no se materializaría de forma inmediata, y a partir de este momento se encargaría de ella la referida Junta de la gestión de los propios de Madrid; la cual, tras casi dos años de ejercicio, elevaba un memorial o representación al Concejo -presentada en el pleno de 22 de marzo de 1746-, en la que exponía

lo acaecido en la administración de ellos en los 20 meses que los a manejado y los motibos que ymposiblitan suplirse la mayordomía con solo la continuazión de la Junta, por lo que hacía formal dimisión de la administración de dichos propios para que Madrid nombrase mayordomo de ellos. ${ }^{6}$

\subsection{Iniciación}

En virtud de este hecho, el Consistorio convocaba una nueva reunión, a la postre celebrada el 24 de marzo, para volver a visualizar la citada representación, junto a los antecedentes de la instauración de la referida Junta, estipulándose que "para el primer Ayuntamiento pasadas las próximas bacaciones se llame para nombrar mayordomo de propios". A partir de ese momento, cada uno de los pretendientes le dirigía al Ayuntamiento una súplica donde

5 A.V.M., Secretaria, 3-29-84. A.V.M., Secretaría, libros de acuerdos, núm. 170 
defendían la idoneidad de sus candidaturas en virtud de los méritos y valores que atesoraban. Finalizada la Semana Santa, según lo acordado en la sesión del 24 de marzo, dicho tema sería tratado en el pleno del 18 de abril, cuando se presentarían las diferentes peticiones de los aspirantes (D. Antonio de Bengoa y Arriola, D. Francisco Gutiérrez de Carriazo y D. Gaspar Antonio del Campo). Asimismo, al unísono se mostró una representación del contador Francisco Verdugo, encargado de la liquidación y fenecimiento de las cuentas de la mayordomía del cargo del fallecido Eusebio de Sabugal, en la que se daba cuenta de la conclusión únicamente de las relativas al periodo comprendido entre 1 de enero de 1722 y diziembre de 1728, faltando las sucesivas hasta abril de 1744 .

\subsection{Suspensión cautelar}

Finalmente, el Concejo decidía, en atención a lo anteriormente expuesto, suspender el proceso de selección, y volvía a encomendar la administración de los propios a la "Junta diputada para la dirección y administración de dichos propios". Estas actuaciones dejaron constancia escrita por triple partida: la primera, en una anotación del secretario al margen de la súplica, reflejo de lo tratado en la sesión del Concejo; la segunda, en forma de acuerdo contenido en el correspondiente libro de actas, y la tercera representada en un copia simple del mismo:

Haviendo precedido llamamiento antediem para nombrar mayordomo de propios de esta Villa conforme a lo acordado en veinte y quatro de marzo próximo se vieron los memoriales presentados por el señor don Antonio de Vengoa, don Francisco Gutiérrez Carriazo y don Gaspar Antonio del Campo pretendiendo cada uno les confiera Madrid la referida mayordomía de propios que últimamente sirvió don Eusebio de Sabugal y Zepeda, de cuya nominación fianzas y demás antecedentes se hizo igualmente relación y assí mismo se vio una representación del contador don Francisco Verdugo, su fecha este día, en que expone que en obediencia de lo acordado por Madrid y cumpliendo con las órdenes que se le han comunicado tiene liquidadas, glosadas y fenecidas, de oficio y sin asistencia de la parte, las quentas de la referida mayordomía de propios del cargo del dicho don Eusebio de Sabugal correspondientes a siete años desde primero de henero de mil setecientos y veinte y dos hasta fin de diziembre de mil setecientos y veinte $\mathrm{y}$ dos hasta fin de diziembre de mil setecientos y veinte y ocho, cuyo cargo data y resulta se expresa y que las subcesivas quentas de hasta fin de abril de mil setecientos y quarenta y quatro que el dicho don Eusebio cesó en en (sic) este encargo y se hallavan para ponerlas en estado de glosa en poder de don Agustín Gómez 
Lozano éste las havía pasado al de el dicho contador para ejecutar lo que con las antecedentes, en cuyo trabajo está entendiendo para fenezerlas con la brevedad que pide su importante conclusión y acreditar por este medio su deseo de sacrificarse en servicio de Madrid.

Salió el señor don Joseph de Olivares.

$\mathrm{Y}$ tratado y conferido sobre ambos asumptos en vista de todo lo referido se acordó de conformidad suspender como se suspende por ahora el nombramiento de mayordomo de propios de esta villa mediante los motibos que Madrid ha tenido oy presentes para estimarlo así por conveniente. Y al tiempo que se haya de hazer la nominación se traiga la Concordia en que se prescrive la forma que debe observase en la distribucción de este y otros empleos y la junta diputada para la dirección y administracción de dichos propios continue también por ahora en este encargo con el zelo y eficacia que Madrid a experimentado y fía de su conducta.... ${ }^{8}$

Con todo dicha suspensión sólo tenía que ser temporal, en concreto, hasta finales del mes de mayo, según consta en un acuerdo de 9 del mismo mes y en su correspondiente copia simple:

Haviéndose tratado y conferido en este Ayuntamiento sobre la importante providencia de nombrar persona que sirba la mayordomía de propios de esta villa como medio más eficaz y proporcionado a la mejor administrazión y utilidad de ellos se acordó que la suspensión de este nombramiento acordada, con la calidad de por ahora, en el ayuntamiento de diez y ocho de abril próximo passado se entienda y corra tan solamente hasta fin de este presente mes. ${ }^{9}$

\subsection{Iniciación (subsanación)}

Sin embargo esto no llegó a ser del todo cierto, pues el Concejo no se reunió para tratar el tema hasta el 1 de julio. No obstante, la actuación del Consistorio se limitó en ese día a establecer el salario que debía corresponderle al mayordomo, unos 6.600 reales de vellón anuales, que podían verse incrementados con el "diez por ciento de la renta que cobrase de todos los efectos que descubriese, aumentase y pusiese corrientes a beneficio de esta villa" ${ }^{10}$ Asimismo se estipuló la obligación del mayordomo a presentar fianzas antes de la toma de posesión del cargo por un valor de

8 A.V.M., Secretaría, 3-29-85. A.V.M., Secretaría, 1-319-1. A.V.M., Secretaría, libros de acuerdos, núm. 172.

9 A.V.M., Secretaría, 3-29-85. A.V.M., Secretaría, 1-319-1. A.V.M., Secretaría, libros de acuerdos, núm. 172.

10 A.V.M., Secretaria, 3-29-85. A.V.M., Secretaría, 1-319-1. A.V.M., Secretaría, libros de acuerdos, núm. 172 . 
doze mil ducados en hipotecas dentro del casco de esta villa a satisfacción de Madrid, vien entendido que a proporción de lo que en adelante creciese la renta anual de dichos propios y porque le ba asignado el diez por ciento ha de dar y aumentar las fianzas correspondientes, ${ }^{11}$

con el fin de asegurar la mayordomía y con ella los propios de Madrid de posibles irregularidades, alcances, etcétera.

Por último se encomendó al secretario del Ayuntamiento la tarea de comunicar las citadas premisas a cada uno de los pretendientes, quienes tenían que dar respuesta oportuna al citado secretario para su posterior participación al Consistorio.

Al poco tiempo, concretamente el 6 de julio, el Concejo acordó volver a reunirse el día 11 con el fin de proceder al nombramiento, sin embargo, dicho acto acabaría posponiéndose para el día 18. En esta jornada se haría presentación, en forma de súplica, de las diferentes respuestas de los candidatos a lo solicitado en el referido pleno de 1 de julio, incluyéndose, al parecer, la de un nuevo pretendiente, Gonzalo Hurtado de Mendoza y Figueroa. Una vez vistos los memoriales por los miembros del Consistorio, se llegó a un doble acuerdo. En primer lugar se accedió a las pretensiones de Antonio de Bengoa y Arriola, quien tras una breve relación de sus méritos, notificaba su renuncia a optar al servicio de dicho empleo, pues

haviéndosele echo saver lo acordado por vuestra señoría ilustrísima últimamente en asumpto de las zircunstanzias con que ha de entrar a servir dicho empleo el que nuevamente se eliga...desiste desde luego de ella. ${ }^{12}$,

en razón de lo cual requería la devolución de la instancia que había presentado en un principio con dicho fin, además de solicitar "que no conste por escrito cosa alguna de haver sido pretendiente el suplicante a el menzionado empleo".

En el siguiente acuerdo se citaban las propuestas del resto de aspirantes, destacando la de Gaspar Antonio de Campo, único candidato dispuesto a aceptar las condiciones estipuladas. En función de esto se recordaba de nuevo que únicamente podrían acceder al cargo quienes se ajustasen a los requisitos convenidos por el Concejo el referido primero de julio. Hecho éste que motivó la pronta respuesta de Gonzalo Hurtado de Mendoza y Figueroa, quien elevó una súplica al Ayuntamiento indicando que "está llano a dar la

11 A.V.M., Secretaria, 3-29-85. A.V.M., Secretaría, 1-319-1. A.V.M., Secretaría, libros de acuerdos, no. 172.

12 A.V.M., Secretaria, 3-29-85. 
fianza y lo demás que tubiere V.S.I. por combeniente para la mejor seguridad de los caudales". Por otro lado, en el caso de Gaspar Antonio del Campo se encargaba al secretario del Consistorio el cumplimiento de lo contenido en la Sentencia de Montalbo y en la Concordia de Bobadilla. Es de suponer que se estaban refiriendo a la obligación del pretendiente a presentar documentos que demostrasen su "limpieza e hidalguía de sangre", pues días después, concretamente el 22 de julio, el mencionado Gaspar Antonio remitió un oficio a Vicente Francisco Verdugo, secretario del Ayuntamiento, en el que comunicaba su predisposición a entregar dichos papeles "en toda la semana próxima”. Fiel a su palabra envió una petición acompañada de diversas actas notariales, algunas de las cuales incluían numerosas partidas de bautismo y casamiento, además de extractos de libros de matrícula y padrones.

\subsection{Suspensión indeterminada}

Sin embargo, cuando todo apuntaba a la elección definitiva de un nuevo mayordomo, Fernando VI decidió suprimir la figura del corregidor en Madrid, creando en su lugar el Gobierno Político y Militar, que, en manos del conde de Maceda, asumió un gran número de competencias, entre ellas la gestión de los propios; razón ésta que debió de paralizar y posponer dicho nombramiento.

\subsection{Levantamiento de suspensión}

No obstante, meses después, el Concejo se reunía, el 5 de julio de 1747, para decidir el modo de ingreso de los caudales de propios en el arca de la Tesorería de Causa Pública que iba a establecer Fernando VI en la villa de Madrid, acordándose que

respecto de que ha de haver mayordomo de propios que....administren y recauden con la orden y acuerdo del Aiuntamiento todas las tierras, zensos, sotos y casas y demás hazienda y propiedades de la villa, éste en cumplimiento de su empleo ponga mensualmente en la dicha Thesorería de Causa Pública al fin de cada mes las cantidades que en él huviese recaudado de la expresada administrazión de propios. ${ }^{13}$

De igual modo, el Consistorio resolvió el 30 de agosto, ante la proximidad de la festividad de san Miguel, "en que es establezimiento, ley y concordia con 173. 
los caballeros escuderos de esta villa el nombrarse mayordomo de propios", ${ }^{14}$ elevar un escrito al Gobernador Político y Militar de Madrid para que accediese a dicho nombramiento en atención a lo tratado en la sesión del 5 de julio. Así, con dicho fin, el 11 de septiembre se expedió un memorial -conservado en forma de original y borrador-, que, junto a una copia autenticada del citado acuerdo de 5 de julio fue enviado al Conde de Maceda.

La respuesta llegaría de manos del teniente de gobernador, Felipe de Solís y Gante, quien, a modo de intermediario, dirigió al secretario del Concejo, Julián Moreno Villodas, un escrito del Gobernador, fechado el 22 de septiembre de 1747 -adjunto a un oficio de remisión del mismo día-, mediante el cual solicitaba que se le entregase

el estatuto, privilegio o concordia que tenga para este nombramiento y copia del que últimamente hizo y que me informe quién fue el último que sirvió este empleo y bajo qué reglas y quáles fueron las que se observaron después de su muerte. ${ }^{15}$

Finalmente, el Conde de Maceda accedió a las pretensiones del Ayuntamiento de Madrid, según consta en una nota al margen de la referida representación del 12 de octubre, que fue devuelta al Consistorio, dándose cuenta de la misma en el pleno del día siguiente:

Hízose presente la resolución de S.E. en decreto de 12 de este mes a la representación de Madrid de 11 de septiembre proxsimo sobre la nominación de mayordomo de propios a fin de elegirle el Ayuntamiento en fuerza de lo prebenido en la Sentencia del señor Alfonso Díaz de Montalbo y Concordia del señor Juan de Bobadilla por ser empleo correspondiente a los cavalleros hijosdalgo de esta Villa, mandando S.E. se hiciese como Madrid lo proponía en dicha representazión y acuerdo de cinco de julio de este año que la acompañaba y que en su consecuencia nombrase mayordomo de propios. Y se acordó se llame para ber dicha resolución el martes 17 del corriente.

(Acuerdo. Madrid, 13 de octubre de 1747).

Fiel a lo dispuesto en el acuerdo anterior, el Concejo volvía a reunirse el 17 de octubre, y dispuso lo siguiente:

Para nombrar la mayordomía de propios que se halla vacante y la thenenzia de maestro mayor de Madrid se llame a el Ayuntamiento el viernes próximo como

14 A.V.M., Secretaría, 1-317-6. A.V.M., Secretaría, libros de acuerdos, núm. 173.

15 A.V.M., Secretaría, 3-29-87. 
subzesibamente para proseguir en las elecciones del día de san Miguel que están por executar.

(Acuerdo. Madrid, 17 de octubre de 1747).

\subsection{Suspensión cautelar}

Con todo, un acontecimiento inesperado vino a postergar otra vez el nombramiento: la dimisión del Gobernador Político y Militar de Madrid. El Conde de Maceda expedía el 16 de octubre un oficio, que era presentado ante los miembros del Consistorio al día siguiente, en el que daba cuenta de su dimisión al frente del Gobierno. Aunque esta decisión no supuso en un primer momento alteración alguna del calendario marcado para la elección del nuevo mayordomo, posteriormente, en el pleno del 20 de octubre se decidió suspender dicho acto hasta que -una vez restituido el cargo de corregidor en Madrid-, Antonio Heredia y Bazán se pusiera al frente del corregimiento. Asimismo, se decidió, mientras tanto, delegar la gestión de dichos propios en sendos comisarios: Francisco Antonio Zapata y Juan Joaquín de Novales. ${ }^{16}$ Por último, Antonio Heredia y Bazán juraba el 1 de diciembre el citado ministerio ante el Concejo, acordándose en un pleno posterior de 26 de enero "que para el lunes 5 del mes próximo de febrero se llame para tratar y resolber lo más comveniente sobre la nominación de mayordomo de propios y mejor administración y recaudazión de ellos". ${ }^{17}$

\subsection{Iniciación (subsanación)}

Llegado el día no se hizo mención alguna al tema, por lo menos en lo que se refiere a lo deliberado en el pleno de esa jornada, y hubo de esperar hasta el 21 de marzo para ver de nuevo tratada esta cuestión en el Ayuntamiento. En primer lugar

se hizo relazión de los acuerdos, ynformes y demás papeles tocantes a la jubilazión que se conzedió a don Eusebio de Sabugal, mayordomo que fue de dichos propios, formazión de la Junta y demás providenzias que Madrid estimó por combeniente para la administración ynterina de ellos, ${ }^{18}$

y se centra a continuación en disponer una serie de "reglas y nominaziones" para su correcta administración, dada la delicada situación en la que se

16 A.V.M., Secretaría, 1-317-6. A.V.M., Secretaria, libros de acuerdos, núm. 173.

17 A.V.M. Secretaría, 1-322-5. A.V.M., Secretaría, libros de acuerdos, núm. 174.

18 A.V.M., Secretaria, 3-29-88, A.V.M. Secretaría, 1-322-5. A.V.M., Secretaría, libros de acuerdos, núm. 174. 
encontraban, a la vez que destaca en este sentido los puntos: primero ("Madrid ha de nombrar mayordomo de propios a cuio fin se dará llamamiento antediem para después de la inmediata Pasqua de Resurección"19), y décimo ("El mayordomo de propios que Madrid nombrare haya de ser obligado a dar fianzas hasta en cantidad de quatro mil ducados de vellón”). Esta rebaja sustancial en la cantidad a entregar, unos 8.000 ducados, animó a un elevado número de aspirantes a presentar sus solicitudes. Sirva de ejemplo el caso de Alejandro Herrero, quien decía

que haviendo savido estaba vaco el empleo de maiordomo de propios y V.S.I. pasó al oficio del secretario de Ayuntamiento don Vicente Berdugo, en donde hecho cargo de las nueve providencias que con tanta reflexión V.S.I. havía arreglado para establecer una favorable administrazión de ellos y de la fianza de hasta quatro mill ducados dio memorial solicitando dicho empleo. ${ }^{20}$

Incluso hubo quien, como Antonio de Bengoa, renunció a su cargo de regidor en el Ayuntamiento de Madrid para poder entrar en el proceso de selección, según consta en la correspondiente acta notarial.

En esa línea, el 26 de abril se continuó con el nombramiento y se procedió en primer lugar, a examinar las instancias de los distintos candidatos (Antonio de Bengoa, Francisco Gutiérrez de Carriazo, José Joaquín Ballesteros y Sabugal, Gonzalo Hurtado de Mendoza, Mateo de Berrio y Mendía, Alejandro Herrero, Francisco Xaviea de Iruegas Barcena y Alfonso de Castro y Villasante), para advertirse que

algunos de estos no se hallan aprovados y admitidos por Madrid para el goce de los oficios honoríficos ni presentado con sus memoriales papeles algunos que conduzcan a justificar su limpieza e hidalguía de sangre. ${ }^{21}$

Este hecho motivó la suspensión temporal del referido proceso hasta la consecuente subsanación por parte de los candidatos de dicha falta, "a cuyo fin se les asigna por término preciso y peremptorio diez días siguientes a éste". 22 Asimismo se encargaba a Vicente Verdugo la notificación a cada uno de los aspirantes del referido acuerdo para su fiel cumplimiento, pues una

19 A.V.M., Secretaría, 3-29-88, A.V.M. Secretaría, 1-322-5. A.V.M., Secretaría, libros de acuerdos, núm. 174.

20 A.V.M., Secretaría, 3-29-88.

21 A.V.M., Secretaría, 3-29-88, A.v.M. Secretaría, 1-322-5. A.V.M., Secretaría, libros de acuerdos, núm. 174 .

22 A.V.M., Secretaría, 3-29-88, A.v.M. Secretaría, 1-322-5. A.v.M., Secretaría, libros de acuerdos, núm. 174. 
vez finalizado dicho plazo se procedería a convocar al Consistorio para continuar con la elección. Dicho requerimiento obtendría la pronta respuesta de personajes como Alejandro Herrero, quien pedía que, "respecto de tener sus gozes en los reynos de Aragón y Valencia y ser tan limitado el tiempo prefinido que no es posible traerlos en dicho tiempo", se le admitiese a la elección de dicho empleo; o José Ballesteros y Sabugal, quien elevaría al corregidor una súplica, en la que argumentaba las razones por las que no había presentado la documentación solicitada:

se le ha hecho saber por el señor don Vizente Verdugo acuerdo de V.S.I. en que manda que los pretendientes de la maiordomía de propios presenten sus papeles de nobleza en el estado que los tengan y teniéndolos el suplicante corrientes y recivida la informazión en esta Corte con citazión de el señor procurador general; y dado este traslado no ha llegado el caso de evaquarse por no haver podido el suplicante adquirir el goze de su raiz, sin embargo de que en lugar de este tiene en dichos papeles una informazión de nobleza hecha a pedimento de don Diego Joseph Vallesteros, su padre, en la villa de Oliva immediata a la de la Higuera de Fregenal en el mes de junio de mil seiscientos noventa y nuebe; y aunque ha solicitado y está solizitando traher el dicho goze para corroborar dicha ynformazión no lo ha podido hasta aora lograr a causa de no tener conozimiento en aquella tierra y de que por ser frontera de Portugal están mui deshordenados los archivos por los yncendios que repetidas vezes han padezido con las guerras entre el dicho reyno de Portugal y el de España sin embargo de no cesar de solizitarlo junto con la certificazión de el ávito de Santiago que tiene noticia tubo Juan José Ballesteros, hermano de Blas Ballesteros, bisabuelo de el suplicante... ${ }^{23}$

\subsection{Finalización}

De regreso a la jornada del 26 de abril, después de examinarse la solicitudes de los aspirantes, se dilucidó a renglón seguido "si la elección de dicho mayordomo se havía de ejecutar por votos secretos o públicos”, y se inclinó el corregidor, conforme a lo practicado en ocasiones anteriores -pese a la oposición de la mayoría de los capitulares-, por esta última opción. Esta polémica decisión provocó el descontento de regidores como Francisco de Milla, quien argumentó que

respecto de ser esta declarazión depuesta a lo espuesto por la mayor parte de los cavalleros capitulares en este Ayuntamiento protextaba la elección en la forma 
que el señor correxidor havía resuelto y pedía a su señoría $<$ mandase $>$ se le diese por zertificazión, ${ }^{24}$

a lo que accedió el corregidor.

En esa línea, resultan de interés las críticas vertidas por Francisco de Milla contra la decisión adoptada por el Marqués de Rafal, pues abriría una nueva vía procedimental, que se traduciría en diversos documentos. No en vano, el día 29 era presentada de manos del secretario concejil una minuta de certificación solicitada por Francisco de Milla ante el pleno del Concejo para su aprobación, lo que se producía sin mayores impedimentos. Una vez este documento en poder del citado capitular lo remitió junto a un recurso al Consejo, y obtuvo como respuesta un escrito que se envió al marqués de Rafal, según se desprende de un oficio de 13 de mayo del citado corregidor en el que solicitaba a Vicente Verdugo

una certificazión con inserzión del acuerdo de Madrid de 26 de abril próximo pasado executado sobre nombramiento de mayordomo de propios y otra de haverse hecho siempre estas nominaziones por votos públicos,

para enviarlas al Consejo, pero sólo ha llegado hasta nosotros el borrador de la segunda. Finalmente el secretario del Consejo dirigía un oficio, el 25 de mayo, al ayuntamiento madrileño en el que

teniendo presente lo imformado por el señor Marqués de Rafal y expuesto por el señor fiscal ha acordado que, sin embargo del recurso del expresado don Francisco de Milla proceda V.S. a hacer por votos públicos la elección de tal maiordomo de sus propios. 25

De esta resolución se daría cuenta en el pleno del 27 de mayo.

Por último, el pleno del Concejo era convocado para el 3 de julio, previo llamamiento antediem, con objeto de elegir al nuevo mayordomo. Ese día como anticipo al acto de nombramiento se volvían a leer los memoriales de los pretendientes, además de los acuerdos de 21 de marzo y 26 de abril, junto a la resolución del Consejo de 25 de mayo y a las disposiciones al respecto contenidas en la Sentencia de Montalbo y Concordia de Bobadilla. De igual forma, se vieron otros documentos que habían entregado los aspirantes, v.gr. la escritura de renuncia que otorgó Antonio de Bengoa el 25 de abril y diversos

24 A.V.M., Secretaría, 3-29-88, A.V.M. Secretaría, 1-322-5. A.V.M., Secretaría, libros de acuerdos, núm. 174.

25 A.V.M., Secretaria, 3-29-88. 
diplomas de carácter probatorio que fueron presentados para demostrar su nobleza y la de sus ascendientes. A continuación, se acordó que únicamente entrasen en suerte las candidatos que hubieran sido aprobados y admitidos

por Madrid al goze de los ofizios honoríficos que corresponden a los caballeros hijosdalgo desta villa y en esta inteligencia y la de concurrir conexión de parentesco con algunos de los pretendientes en los señores don Joseph de Olibares, don Blas Ruiz Vayllo y don Matheo de la Rea se les prebino debían no allarse presentes al nombramiento. ${ }^{26}$

Finalmente se procedió a la votación y resultó elegido Antonio de Bengoa, según consta en el correspondiente acta del pleno, decisión que le fue notificada al día siguiente por el escribano Fernando Nicolás de Tricio:

En la villa de Madrid a quatro días del mes de julio, año de mil setezientos y ocho, yo, el scribano, hize notorio el acuerdo de Madrid que prezede su fecha tres deste mes a don Antonio de Vengoa en su persona, quien haviéndolo oydo y entendido dijo que estimando como debe las honrras que Madrid lo haze en el nombramiento del empleo que le ha conferido de mayordomo de sus propios le azepta en todo y por todo como en el referido acuerdo y en el antezedente que cita, de veinte y uno de marzo próximo, se contiene y está pronto a cumplir con el thenor y calidades que en uno y otro se espresan y en su conformidad otorgará escriptura de obligazión en esta razón a fabor de Madrid y dará la fianza acordada; y a ello se obliga en bastante forma. ${ }^{27}$

Como quedó expresado anteriormente uno de los requisitos para acceder al cargo radicaba en la presentación de una fianza estimada en 4.000 ducados. Así, con dicho fin, Antonio de Bengoa elevaba una petición al Consistorio en la que proponía como garantía la propiedad de un oficio de contador de millones del partido de Ciudad Real, perteneciente a su padre, Sebastián de Madrid y Baiz. Sin embargo, dicha solicitud fue rechazada en el pleno de 10 de julio, según consta en una nota al margen de la súplica. No obstante, seis días después, Antonio de Bengoa volvía a dirigir una petición al Ayuntamiento, en la que presentaba otras fianzas, consistentes en unas casas sitas en la calle de las Huertas, que se estaban reformando, estimadas en 64.240 reales y sobre las que se habían impuesto dos censos alquitar. No obstante, una vez finalizadas las obras, el valor de dichas viviendas se elevaría a unos

26 A.V.M., Secretaría, 3-29-88, A.V.M., Secretaría, 1-322-5. A.V.M., Secretaría, libros de acuerdos, núm. 174. 
90.000 reales, según constaba en una escritura de tasación del 14 de mayo realizada por el maestro de obras y alarife Francisco Álvarez Buelta. ${ }^{28}$ Además, también ofrecía "otros títulos de pertenencia de diferentes tierras, casa de labor con todas sus oficinas en el lugar de Carabanchel", ${ }^{29}$ propiedad al igual que las anteriores de Julián Fernández Palomo.

Esta instancia era vista en el Consistorio el 17 de julio que decidió encargar al procurador general el cometido de informar sobre el contenido de la misma. El resultado de dicha labor se tradujo en un informe que Antonio Gaspar de Pinedo, a la sazón procurador de Madrid, expidió el 27 de julio. Por otro lado y ante las dudas que dichas propiedades pudieran generar en cuanto a su verdadero valor, Antonio de Bengoa remitió al Concejo una súplica adjunta a una fe notarial del estado y tasación de las obras que se estaban realizando en las casas de la calle de las Huertas. Reunida toda esta documentación se examinó en el Ayuntamiento el día 29 de julio y provocó el recelo de diversos capitulares ante la referida propuesta, si bien el procurador general:

expresó in boze haverle asegurado don Antonio de Vengoa se concluiría con brebedad la obra que en dicha casa de la calle de las Huertas se estaba executando como también la redempzión de los dos censos al quitar sobre ellas impuestos mediante que para ambos fines tenía pactado entregar al dicho Julián Fernández Palomo quatro mil ducados de vellón que havían de quedar a censo sobre las mismas casas y que a quenta de esta cantidad y para prosecuzión de la obra le tenía satisfechos mil y duzientos ducados de vellón por cuio medio parezía se verificaba más indegnizada y segura la hipoteca destas casas para la referida fianza. ${ }^{30}$

Finalmente se dispuso que dichas fianzas serían admitidas con la condición de presentar para finales del mes de septiembre una declaración del maestro de obras si dieran cuenta de la finalización de las obras en las casas de la calle de las Huertas y del valor de las mismas, junto a las escrituras de redención de los dos censos que estaban impuestos sobre ellas, "como también la imposizión de él de los quatro mil ducados en fabor del mismo don Antonio de Vengoa".

28 A.V.M., Secretaria, 3-107-71.

29 El Archivo de Villa conserva sendas escrituras de tasación de dichas propiedades realizadas por el maestro mayor y alarife de Madrid, Felipe de Hueva, el 9 de mayo del año 1742, A.V.M., Secretaría, 3-107-71.

30 A.V.M., Secretaria, 3-29-88. 


\subsection{Ejecución}

El escribano Lorenzo García Hurtado notificó al día siguiente el anterior acuerdo al nuevo mayordomo, quien se aprontó a su cumplimiento. En consecuencia el Concejo volvió a reunirse el 9 de agosto, previo llamamiento antediem, para otorgarle poder para el uso y ejercicio del oficio. En esta jornada se hicieron presentes, en primer lugar, sendas escrituras, ${ }^{31}$ una otorgada el 5 de agosto por Antonio de Bengoa, en la que se obligaba a desempeñar correcta y puntualmente las diferentes funciones del cargo, y otra expedida por Julián Fernández Palomo "su fiador hasta en la cantidad de los quatro mil ducados con las hipotecas admitidas por acuerdo de Madrid de veinte y nuebe de julio", concediéndosele a continuación el poder ${ }^{32}$ necesario para la cobranza de todas las rentas de los propios de Madrid. De este último acto tenemos constancia a través del correspondiente acuerdo del pleno y de la escritura de poder general: ${ }^{33}$

Haviendo precedido llamamiento antediem a todos los caballeros capitulares que están en esta villa y zertificado los porteros de este ayuntamiento haverle dado el señor secretario don Vizente Francisco Verdugo, hizo presentes las dos scripturas otorgadas en 5 de este mes, una por don Antonio de Vengoa, con obligación de su persona y vienes para seguridad de la mayordomía de propios de esta villa que le está conferida por todo el tiempo que sirbiere este oficio, y la otra por Julián Fernández Palomo, su fiador hasta en la cantidad de 4.000 ducados con las ypotecas admitidas por acuerdo de Madrid de 29 de julio próximo, también por todo el tiempo que el dicho don Antonio de Vengoa ejerciere el referido empleo. Y en su consequencia y de el zitado llamamiento precedido para este efecto se acordó otorgar como con efecto se otorgó por Madrid en este Ayuntamiento poder general según los antezedentes en fabor del dicho don Antonio de Vengoa para que perciba y cobre todas las rentas tocantes y pertenezientes a los propios de esta villa a cuyo otorgamiento fueron llamados y se hallaron presentes por testigos Manuel Calbo, Fernando de Santa María y Joseph de Contreras el menor, porteros de este Ayuntamiento.

(Acuerdo. Madrid, 9 de agosto de 1748).

$31 \mathrm{Al}$ respecto puede verse: A.V.M., Secretaría, 3-107-71.

32 "Constituye el último acto de todo el proceso posesorio. Con su otorgamiento por acuerdo del Concejo se culmina y perfecciona el nombramiento y toma de posesión. La Carta de Poder permite el ejercicio en plenitud del oficio de Mayordomo. Se otorga como hemos visto días después y tras haber prestado fianza”. Corral García, Esteban, El mayordomo de Concejo en la Corona de Castilla (s.XIII-s.XVIII). Madrid: El Consultor de los Ayuntamientos y de los Juzgados, 1991, p. 87. 
Asimismo se acordaba el cese de Miguel de Mendoza al frente de la administración y cobranza de los propios, tarea que había ejercido de forma interina hasta el nombramiento de Antonio de Bengoa. Esta decisión le fue notificada el 12 de agosto.

Llegado el mes de septiembre, según lo acordado, Antonio de Bengoa solicitaría diversas veces al maestro mayor que reconociese, tasase y evaluase la renta que podían producir las casas de la calle de las Huertas, con motivo de la finalización de las obras, y no obtuvo más que escusas. Dicha situación obligó al mayordomo a elevar un escrito en el que solicitaba al corregidor que mandase al teniente de maestro mayor o alarife que tuviera a bien la ejecución de dicho reconocimiento. Esta petición sería examinada en el pleno de 30 de octubre, cuando se acordó que el maestro pasara a reconocer las citadas casas. De este modo, Vicente Francisco Verdugo envió ese mismo día una orden del Concejo al maestro Juan Bautista Saqueti, que tuvo cumplida respuesta el 25 de noviembre en forma de un informe, que se remitió junto con un oficio, según el cual la citada obra estaba practicamente concluida y se valoraba en unos 99.500 reales de vellón.

Meses después, concretamente el 27 de enero de 1749, Antonio de Bengoa presentaba en el Ayuntamiento una súplica, en la que daba cuenta de la imposición de un censo con fecha de 13 de enero por valor de 4.000 ducados sobre las casas de la calle de las Huertas, además de solicitar la admisión de dicha hipoteca como fianza en lugar de la que tenía dada el mencionado Julián Fernández Palomo. Este escrito era visto ese mismo día en el Ayuntamiento, y se acordó que informase el procurador general sobre el asunto; quien una vez examinado el caso argumentó en un oficio de 30 de enero que "si V.I. fuere servido podrá diferir a su pretensión". A su vez, dicho diploma se exponía en el Concejo de 5 de febrero, que disponía que se trajeran todos los antecedentes sobre dicho tema para volverlos a ver en la próxima reunión. De este modo, llegamos al día 14 de febrero, en el que, tras una vez examinados todos los documentos, se acordaba admitir la fianza propuesta por Antonio de Bengoa, en consecuencia de lo cual

se dan por libres todas las hipotecadas y obligadas a la responsabilidad de dicha mayordomía por el precitado Julián Fernández Palomo, pónganse en las scripturas y títulos de dicha pertenencia a éste las notas y desglose necesario como también en la scritura de dicho zenso la glosa correspondiente para que en todo tiempo conste quedar afecto al expresado fin. ${ }^{34}$

34 A.V.M., Secretaría, 3-29-88, A.v.M., Secretaría, 1-317-7. A.V.M., Secretaría, libros de acuerdos, núm. 175. A.V.M., Secretaría, 3-107-71. 
Del cumplimiento de lo dispuesto en último lugar daba cuenta un recibo de Antonio de Bengoa, otorgado el 16 de marzo, por el que se atestiguaba la recepción de la escritura de imposición de censo debidamente glosada. Además, con dicho fin, anteriormente, el día 4 de marzo otorgaba fianza ${ }^{35}$ del referido censo para la seguridad de la mayordomía.

Aunque Antonio de Bengoa volvió a elevar una petición al Concejo meses después, esta vez en razón de la retribución de su salario, el procedimiento se daba por concluido con la remisión del oficio precedente de 16 de marzo.

\section{Documentación}

No cabe duda que un proceso tan dilatado tuvo que redundar necesariamente en la expedición de un gran número de documentos, ${ }^{36}$ que aparecen diseminados en varias unidades archivísticas. Sirva de ejemplo el caso de la documentación relativa a la entrega de las fianzas, reunida en una carpeta aparte. A la hora de clasificarlos hemos preferido reunirlos en tres grandes grupos, documentos de trámite, documentos probatorios y documentos de juicio o informativos, ${ }^{37}$ a sabiendas de que bajo el título primero se encontrarán diplomas resultado de

35 A.V.M., Secretaría, 3-107-71.

36 En razón de la diversidad de tipologías aportadas, pueden resultar de utilidad la consulta de las obras siguientes: Carucci, Paola et altri, Documento y archivo de gestión: diplomática de ahora mismo, Carmona: S\&C ediciones, 1994; Fernández Bajón, María Teresa, "Documentación administrativa: una revisión de las tipologías documentales administrativas comunes", Revista General de Información y Documentación, 1996, 6/2, pp. 67-90; García Miraz, María del Mar et altri, "Una aportación al estudio tipológico de la documentación judicial del Antiguo Régimen: los pedimentos”, Primeras Jornadas sobre metodología para la identificación y valoración de fondos documentales de las Administraciones Públicas, Madrid: Dirección de los Archivos Estatales, 1992, pp. 141-149. García Ruipérez, Mariano, Tipología documental municipal, Junta de Comunidades de Castilla, La Mancha, 2002; Lorenzo Cadarso, Pedro Luis, El documento real en la época de los Austrias (1516-1700), Cáceres: Unex, 2001; La documentación judicial en la época de los Austrias. Estudio archivístico y diplomático, Badajoz: Unex, 1999; Moreno Trujillo, María Amparo, Documentos notariales de Santa Fe en la primera mitad del siglo XVI (1514-1549), Madrid: Fundación Matritense del Notariado, 1988; Ostos Salcedo, Pilar y Pardo Rodríguez, María Luisa (eds.), Estudios sobre el notariado europeo (siglos XIV-XV), Sevilla: Universidad, 1997; Pino Rebolledo, Fernando, Tipología de documentos municipales. Siglos XII-XVII, Valladolid: Universidad, 1991; "El notariado andaluz en el tránsito de la Edad Media a la Edad Moderna”. I Jornadas sobre el notariado en Andalucía, Sevilla: Ilustre Colegio Notarial de Sevilla, 1995; Real Díaz, José Joaquín, Estudio diplomático del documento indiano, Madrid: Dirección de Archivos Estatales, 1991; Rojas Vaca, María Dolores, Una Escribanía Pública Gaditana en el Siglo XVI (1560-1570), Análisis documental, Cádiz: Universidad, 1993; Sierra Valenti, Eduardo, "El expediente administrativo. Esbozo de tipología documental”, Boletín de la ANABAD, 1979, XXIX/2, pp. 66-67.

37 Acerca del concepto de documento pueden consultarse los siguientes trabajos: Galende Díaz, Juan Carlos y García Ruipérez, Mariano, "El concepto de documento desde una perspectiva interdisciplinar: de la diplomática a la archivística”, en Revista General de Información y Documentación, 2003, 13/2, pp. 7-35. Luis Núñez Contreras, "Concepto de documento", en Archivística. Estudios Básicos, Sevilla: Diputación Provincial, 1981, pp. 25-43. 
las distintas fases del expediente: iniciación, tramitación/instrucción, resolución y notificación/ejecución:

\subsection{Documentos de trámite}

Bajo esta denominación recogemos cualquier testimonio escrito producto del concurso de los distintos funcionarios, oficinas del Ayuntamiento y particulares en el desarrollo normal del procedimiento, desde su inicio hasta su conclusión. En esa línea, pueden subividirse de la siguiente manera: Documentos de decisión (auto en acta, llamamiento, orden del Concejo -copia simple- y poder general del Concejo); Documentos de los ciudadanos (peticiones/súplicas, memorial/representación), y Documentos de transmisión (oficio, oficio de remisión).

\subsection{Documentos probatorios}

La función de este tipo de diplomas se reduce a servir de garantía o prueba de la realización, posesión o cumplimiento de algún mérito, circunstancia o requisito solicitado en el procedimiento, lo que perpetua su recuerdo, sin entrar a valorar si fueron escriturados "como prueba o ad probationen de algo existente y ya realizado" 38 o no. Entre los primeros, se encontrarían los documentos denominados de constancia (acuerdo en acta, acta del concejo -borrador-, certificación del secretario del Concejo -copia autenticada-, certificación de llamamiento, acta de notificación de un acuerdo, recibo, fe notarial, acta notarial de solicitud de compulsa de diferentes partidas -bautismo, casamiento, libros de matrícula y padrones- y de respuesta y auto favorable a la petición, acta notarial de notificación de pedimiento y auto, acta notarial de compulsa de partidas de bautismo y casamiento, acta notarial de compulsa de la información contenida en padrones y libros de matrícula). Entre los segundos: fianza, escritura de obligación, escritura de tasación -copia simple-, relación de méritos y escritura de renuncia.

\subsection{Documentos de juicio o informativos}

En ocasiones durante la tramitación podían surgir dudas de carácter procedimental, que eran subsanadas previa petición a la instancia correspondiente de los informes necesarios. Asimismo, en otros casos, antes de iniciarse el

38 Ángel Riesco Terrero, Vocabulario Científico-Técnico de Paleografía, Diplomática y Ciencias Afines, Madrid: Barrero\&Azedo, 2003, p. 127. En relación a este asunto, consúltese: Real Díaz, José Joaquín - Estudio diplomático..., p. 8. 
acto o diligencia en cuestión se procedía a la lectura o consulta, a modo de guía, de diferentes textos de signo orientativo, caso de la Sentencia de Montalvo o Concordia de Bobadilla. Los expedientes analizados conservan un tipo documental de este orden: el informe; que, en opinión de Ángel Riesco Terrero, es un:

documento de contenido y carácter informativo, elaborado y expedido, de oficio o a petición de la autoridad, bien por funcionario público o semipúblico, bien por profesional y persona cualificada, bien por particular ajeno a los conocimientos jurídico-diplomáticos. Va dirigido al rey, a la Corte, al juez o tribunal, a la autoridad competente, dependiendo de su contenido o a una institución u organismo de tipo administrativo, docente, gubernativo, militar, hospitalario, ministerial, etc. Guarda cierta semejanza con el memorial y el parecer, y es de obligado cumplimiento tras las pesquisas, visitas oficiales, juicios de residencia y en asuntos y materias tocantes a la justicia. Cuando el informe procede de persona particular totalmente ajena al mundo del derecho penal y administrativo, o el objeto de la información versa sobre asuntos de otra naturaleza, este tipo documental puede no ajustarse al de oficio en cuanto a estructura y formalidades y hasta resultar confuso e incluir errores.... 39

Los ejemplares que han llegado hasta nosotros fueron redactados por el procurador general ${ }^{40}$ a petición del Concejo.

\section{Conclusiones}

Desde siempre, de manera más o menos tipificada, los actos de la administración estuvieron sujetos a norma. Durante la Edad Media, los documentos, nos darán pistas, a través del análisis de su estructura y cláusulas que los conforman, sobre cómo fueron gestados. Con la llegada de la modernidad se notará un cambio de tendencia, que se traducirá en la necesidad u obligación de conservar y escriturar no sólo el documento resultado de la finalización del procedimiento, sino también cada uno de los testimonios que pudieran

39 Ángel Riesco Terrero, Vocabulario científico-técnico..., p. 209.

40 "Viose un memorial de don Antonio Vengoa en que hacía exibizión de los títulos de pertenenzia de unas casas sitas en la calle de las Huertas y otros raízes en el lugar de Carabanchel de Arriba que posehe Julián Fernández Palomo, escribano del número desta villa, quien estába pronto a afianzar con estas ypotecas la Mayordomía de Propios que por Madrid se ha conferido al dicho don Antonio, que suplicaba se sirbiese admitir esta fianza. Y, vistas asimismo las tres tasaziones que igualmente acompañan dichos títulos por lo respectibo al valor de los espresados efectos, se acordó ynforme el señor Procurador General”, (Acuerdo. 17 de julio de 1748), AVM., Secretaría, 1-322-5. 
producirse al hilo de su normal desarrollo. La búsqueda de una mejor racionalización de la prácticas administrativas, dada su complejidad, acorde con los nuevos tiempos, abogó por la producción y conservación de este tipo de "pruebas". No en vano, habría una preocupación cada vez mayor por el conocimiento y puesta por escrito de las normas que regulaban los actos y actividades de la administración, a fin de evitar conflictos o diferencias de criterio. Sirva de ejemplo de esto último, la polémica suscitada por la forma en que debía realizarse la votación para la elección de mayordomo de propios, que provocó la consecuente queja de Francisco de Milla. Con todo, la mayoría de los procedimientos se realizaban de acuerdo a la costumbre, lo que originaba numerosos problemas; y debía buscarse en caso de duda, la información necesaria entre los papeles del archivo y de las secretarias, para su puesta por escrito. No en vano, las dudas llegaban incluso, en el ámbito de los nombramientos, hasta al tipo documental que debía emplearse en la resolución. Esas cuestiones, son las que permitirán en la Edad Contemporánea, de cara a una mayor transparencia de los actos y defensión de cada una de las partes, al menos en teoría, que el fonema se torne en letra impresa. A partir de este momento, el derecho administrativo, en forma de ley o normativa de otro rango, será quien condicione el estudio de la genésis documental; pues llegará a dividir, caso de la actualidad, el procedimiento en diferentes fases y regulará de modo estricto lo que debe acontecer en cada una de ellas, todo lo cual se plasmará en la estructura del expediente.

Por lo que respecta a nuestra época y al terreno de la documentación municipal, se asistirá a una normalización, pero sin la supuesta rigidez de momentos posteriores. En esa línea puede comprobarse, como en el caso que nos ocupa, que el procedimiento se compone de diferentes subprocedimientos, que cristalizaron en las correspondientes unidades archivísticas, con motivo de los vaivenes de los que fue partícipe, de manera más o menos obligada.

Por último, el hecho de hablar de "procedimientos" en plural, tiene su base en los diferentes "métodos" que se aplicaban conforme al negocio jurídico o asunto del que eran objeto, lo que provocaba modificaciones en su desarrollo, estructura y documentación aportada.

\section{BibliografíA}

Carucci, Paola et altri, Documento y archivo de gestión: diplomática de ahora mismo, Carmona: S\&C ediciones, 1994.

Corral García, Esteban, El mayordomo de Concejo en la Corona de Castilla (s.XIII-s.XVIII), Madrid: El Consultor de los Ayuntamientos y de los Juzgados, 1991. 
Fernández Bajón, María Teresa, "Documentación administrativa: una revisión de las tipologías documentales administrativas comunes", en Revista General de Información y Documentación, 1996, 6/2, pp. 67-90.

Galende Díaz, Juan Carlos y García Ruipérez, Mariano, "El concepto de documento desde una perspectiva interdisciplinar: de la diplomática a la archivística", en Revista General de Información y Documentación, 2003, 13/2, pp. 7-35.

García Miraz, María del Mar et altri, "Una aportación al estudio tipológico de la documentación judicial del Antiguo Régimen: los pedimentos", en Primeras Jornadas sobre Metodología para la Identificación y Valoración de Fondos Documentales de las Administraciones Públicas, Madrid: Dirección de los Archivos Estatales, 1992, pp. 141-149.

García Ruipérez, Mariano, Tipología documental municipal, Junta de Comunidades de Castilla-La Mancha, 2002.

Gibert y Sánchez de La Vega, Rafael, El concejo de Madrid. Su organización en los siglos XII al XV, Madrid, 1949, p. 241.

Lorenzo Cadarso, Pedro Luis, La documentación judicial en la época de los Austrias. Estudio archivístico y diplomático, Badajoz: Unex, 1999.

El documento real en la época de los Austrias (1516-1700), Cáceres: Unex, 2001.

Moreno Trujillo, María Amparo, Documentos notariales de Santa Fe en la primera mitad del siglo XVI (1514-1549), Madrid: Fundación Matritense del Notariado, 1988.

Núñez Contreras, Luis, "Concepto de documento", en Archivística. Estudios Básicos, Sevilla: Diputación Provincial, 1981, p. 25-43.

Ostos Salcedo, Pilar y Pardo Rodríguez, María Luisa (eds.), El notariado andaluz en el tránsito de la Edad Media a la Edad Moderna, Sevilla: Ilustre Colegio Notarial de Sevilla, 1995.

, Estudios sobre el notariado europeo (siglos XIV-XV), Sevilla: Universidad, 1997.

Pino Rebolledo, Fernando, Tipología de documentos municipales. Siglos XII-XVII, Valladolid: Universidad, 1991.

Real Díaz, José Joaquín, Estudio diplomático del documento indiano, Madrid: Dirección de Archivos Estatales, 1991.

Riesco Terrero, Ángel, Vocabulario Científico-Técnico de Paleografía, Diplomática y Ciencias Afines, Madrid: Barrero\&Azedo, 2003, p. 127.

Rojas Vaca, María Dolores, Una Escribania Pública Gaditana en el Siglo XVI (1560-1570). Análisis documental, Cádiz: Universidad, 1993.

Sánchez Albornoz, Claudio, "El Reino Astur-Leonés”, en Historia de España de Menéndez Pidal, Madrid: Espasa Calpe, 1980, vol. VII, pp. 469-473. 
Sierra Valenti, Eduardo, "El expediente administrativo. Esbozo de tipología documental", en Boletín de la ANABAD, 1979, XXIX/2, pp. 66-67.

\section{Fuentes}

Archivo de Villa de Madrid (en adelante A.V.M.), sección Secretaría.

A.V.M., Secretaría, 4-329-41.

A.V.M., Secretaría, 3-29-84.

A.V.M., Secretaria, 3-29-85.

A.V.M., Secretaría, 3-29-87.

A.V.M., Secretaría, 3-29-88.

A.V.M., Secretaria, 1-317-6.

A.V.M., Secretaria, 1-319-1.

A.V.M., Secretaría, 1-322-5.

A.V.M., Secretaría, 3-107-71.

A.V.M., Secretaría, libros de acuerdos, núm. 170.

A.V.M., Secretaría, libros de acuerdos, núm. 172.

A.V.M., Secretaría, libros de acuerdos, núm. 173.

A.V.M., Secretaría, libros de acuerdos, núm. 174. 